\title{
Single $\pi^{0}$ Electroproduction in the Resonance Region with CLAS
}

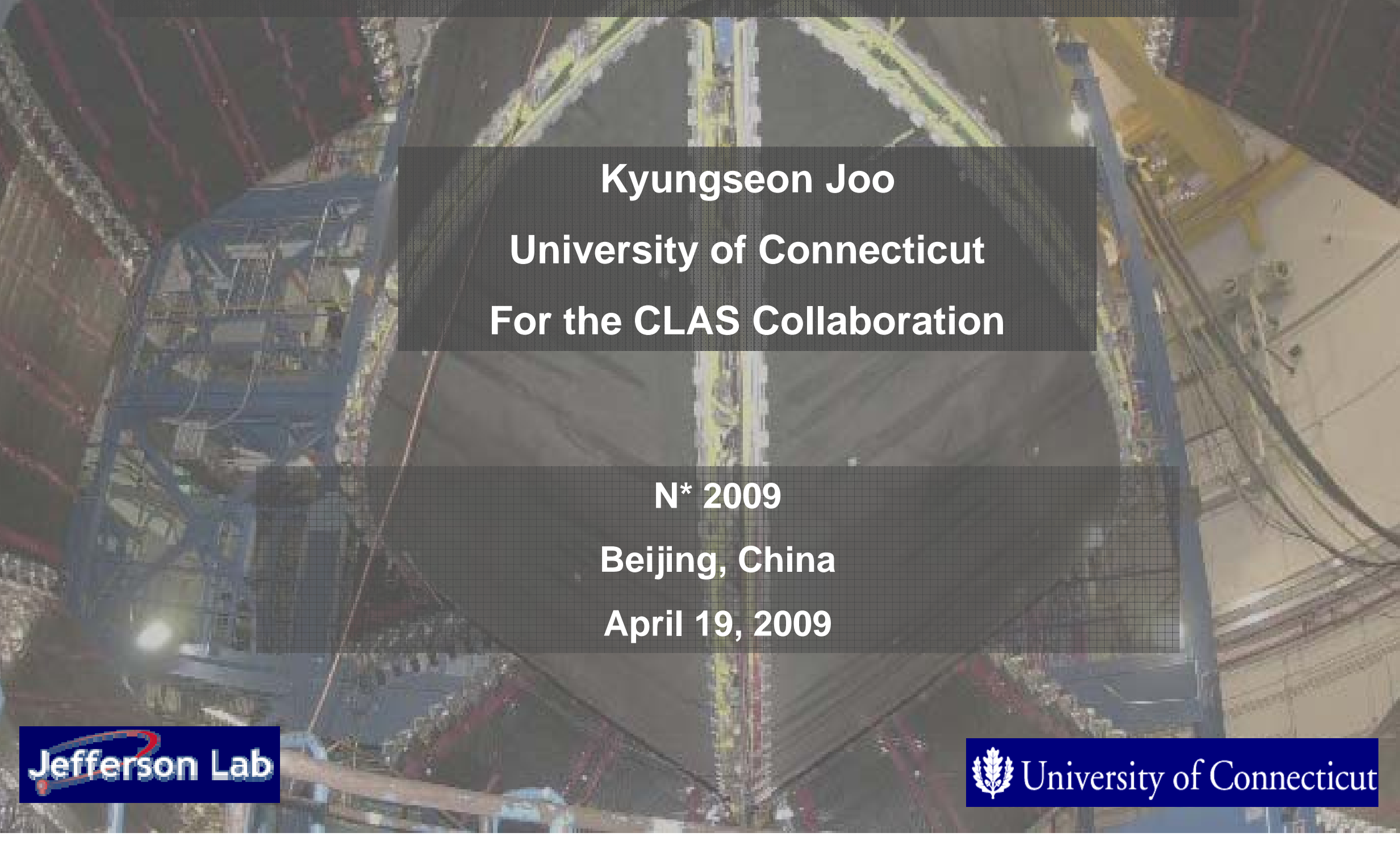




\section{Electromagnetic Excitation of $\mathrm{N}^{*}$}

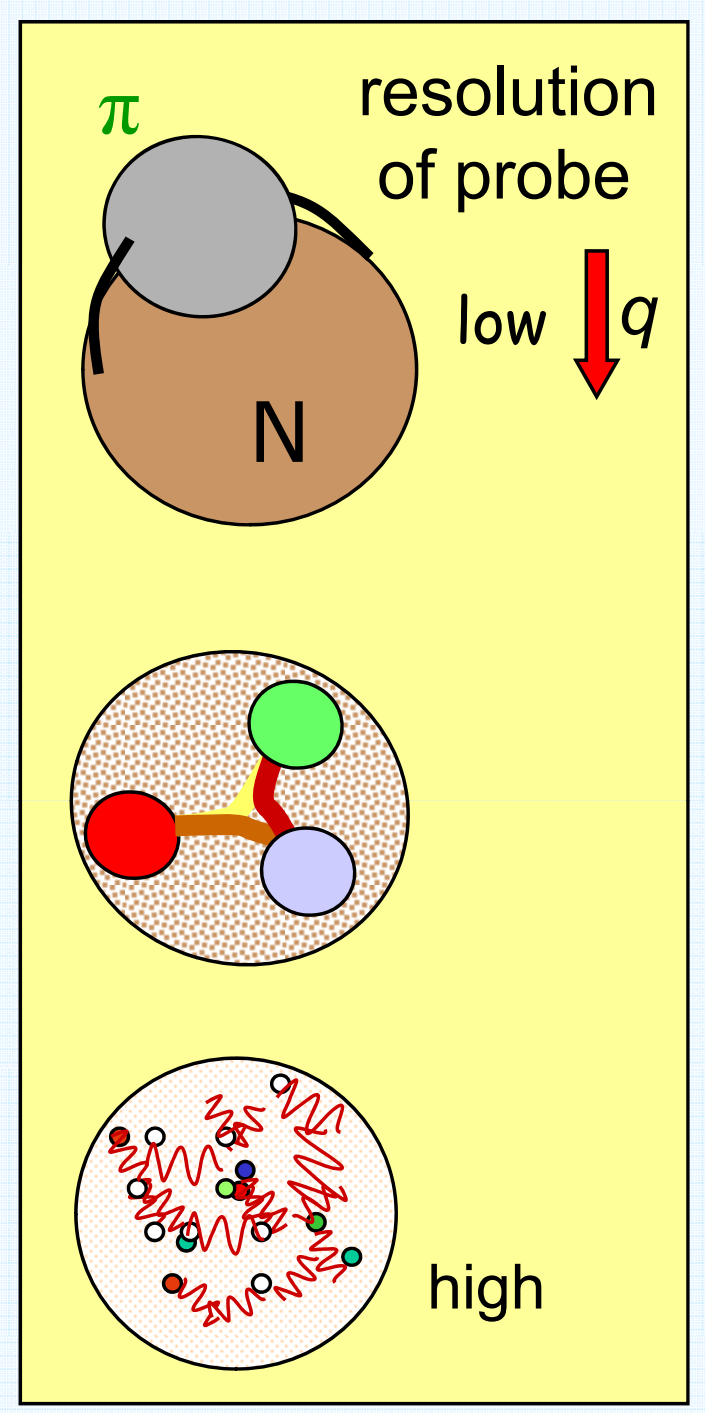

- Allows to address the central question: What are the relevant degrees-of-freedom at varying distance scale? 


\section{Electromagnetic Excitation of $N^{*} s$}

The experimental $N^{*}$ Program has two major components:

1) Accurate measurements of transition form factors $\left(A_{3 / 2}, A_{1 / 21}\right.$ $S_{1 / 2}$ ) of known states as photon virtuality $\left(Q^{2}\right)$ to probe their internal structure and confining mechanism

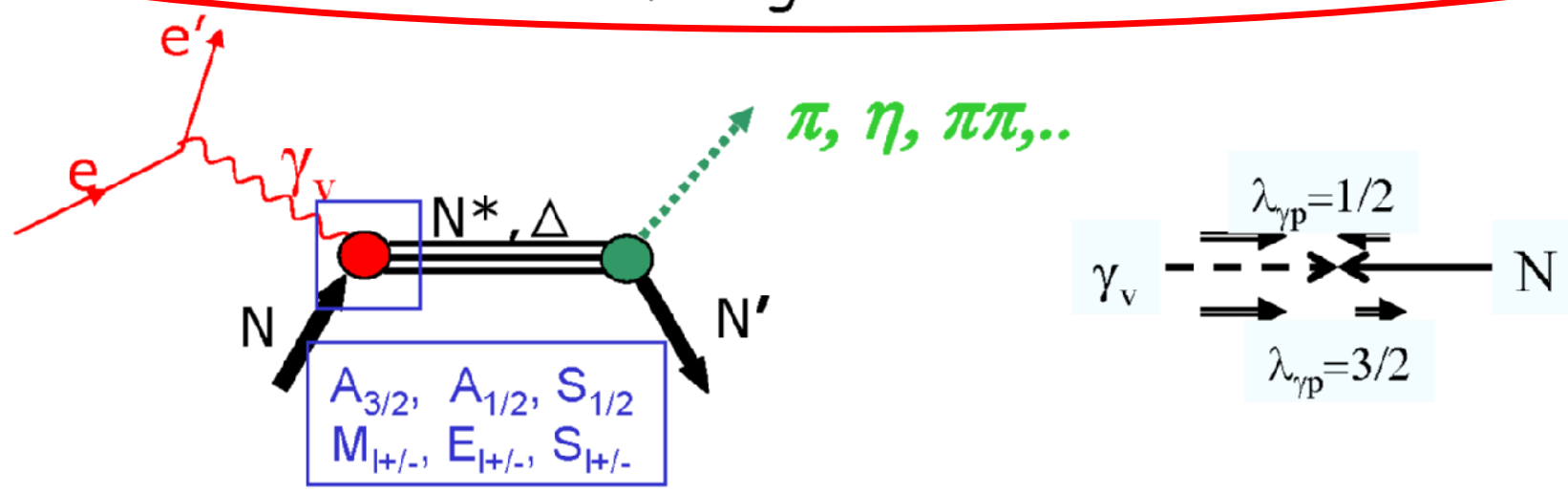

2) Search for undiscovered new baryon states.

Both parts of the program are being pursued in various decay channels, e.g. $N \pi, p \eta, p \pi^{+} \pi, K \wedge, K \Sigma, p \omega, p p^{0}$ using cross sections and polarization observables. 


\section{$\mathrm{SU}(6) \times \mathrm{O}(3)$ Classification of Low Lying $\mathrm{N}^{*}$}

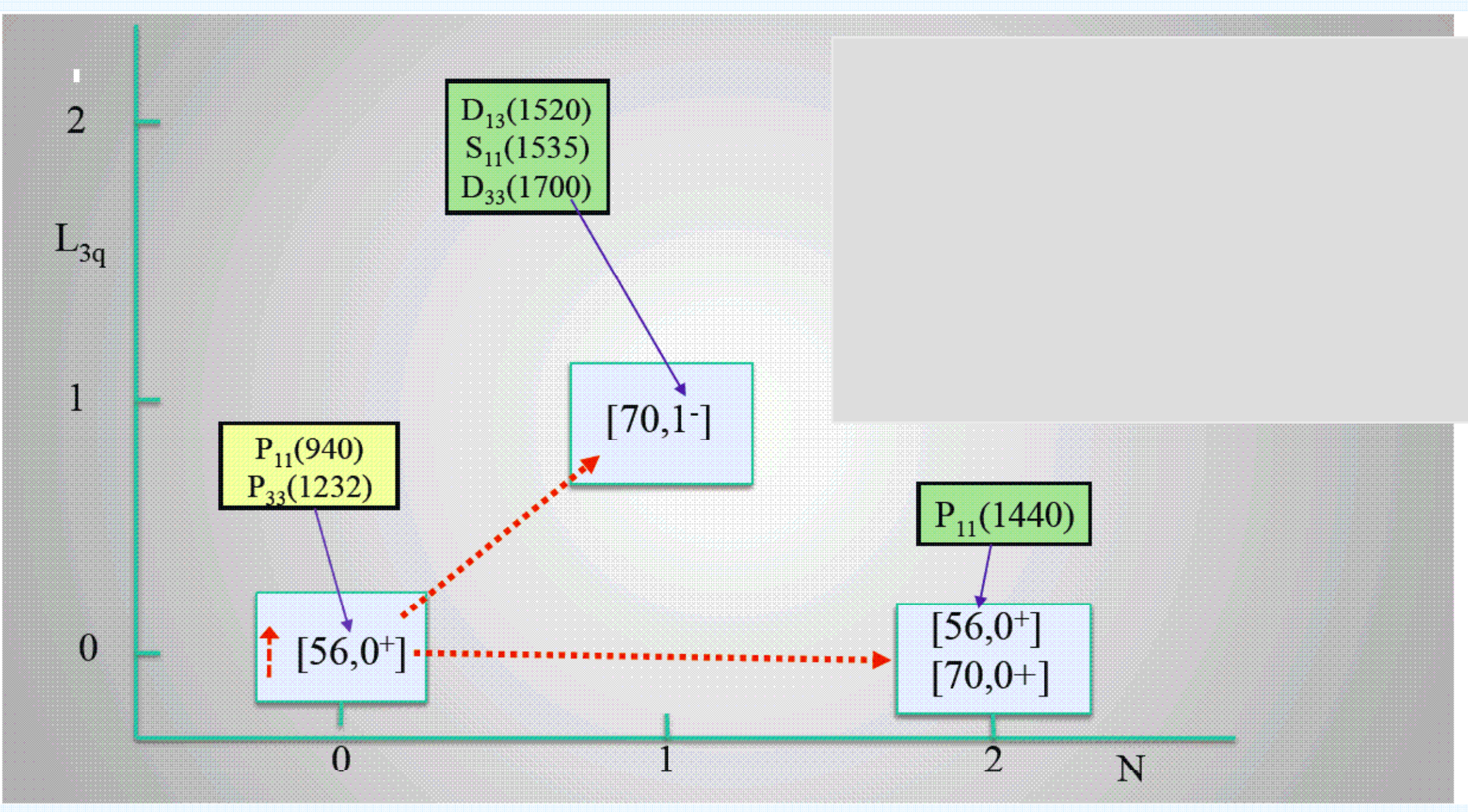




\section{Exclusive Processes in $\mathrm{N}^{*}$ Studies}

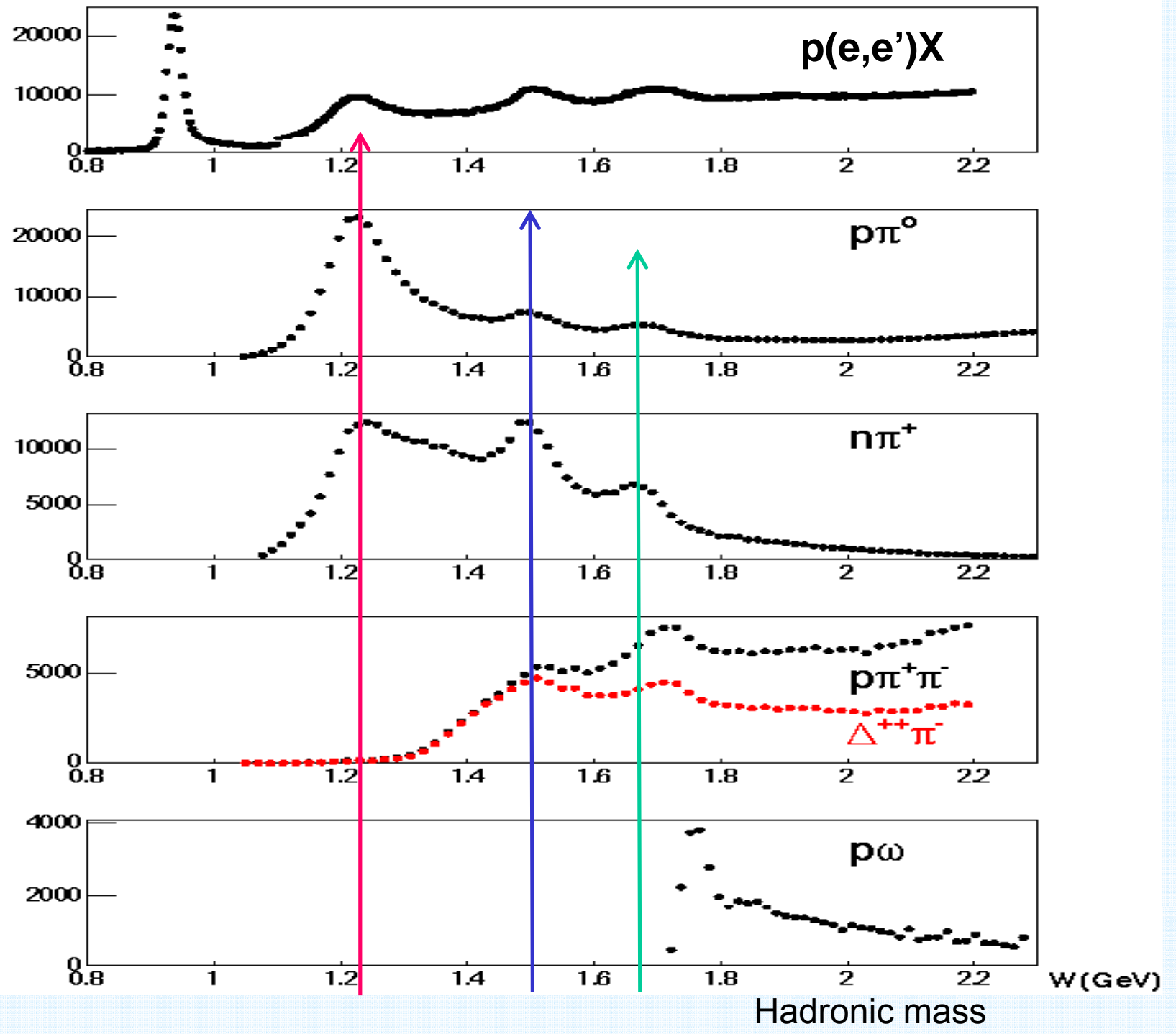




\section{Transition Form Factors of Low Lying N* States}

\section{$p \pi^{0}$ channel is important to study:}

The $\gamma * N \Delta(1232)$ Quadrupole Transition

$\mathrm{N}^{*}$ Transition Form Factors in the 2 nd Resonance Region -

"Roper" $P_{11}(1440), S_{11}(1535), D_{13}(1520)$ 


\section{The $\gamma * N \Delta(1232)$ Quadrupole Transition}
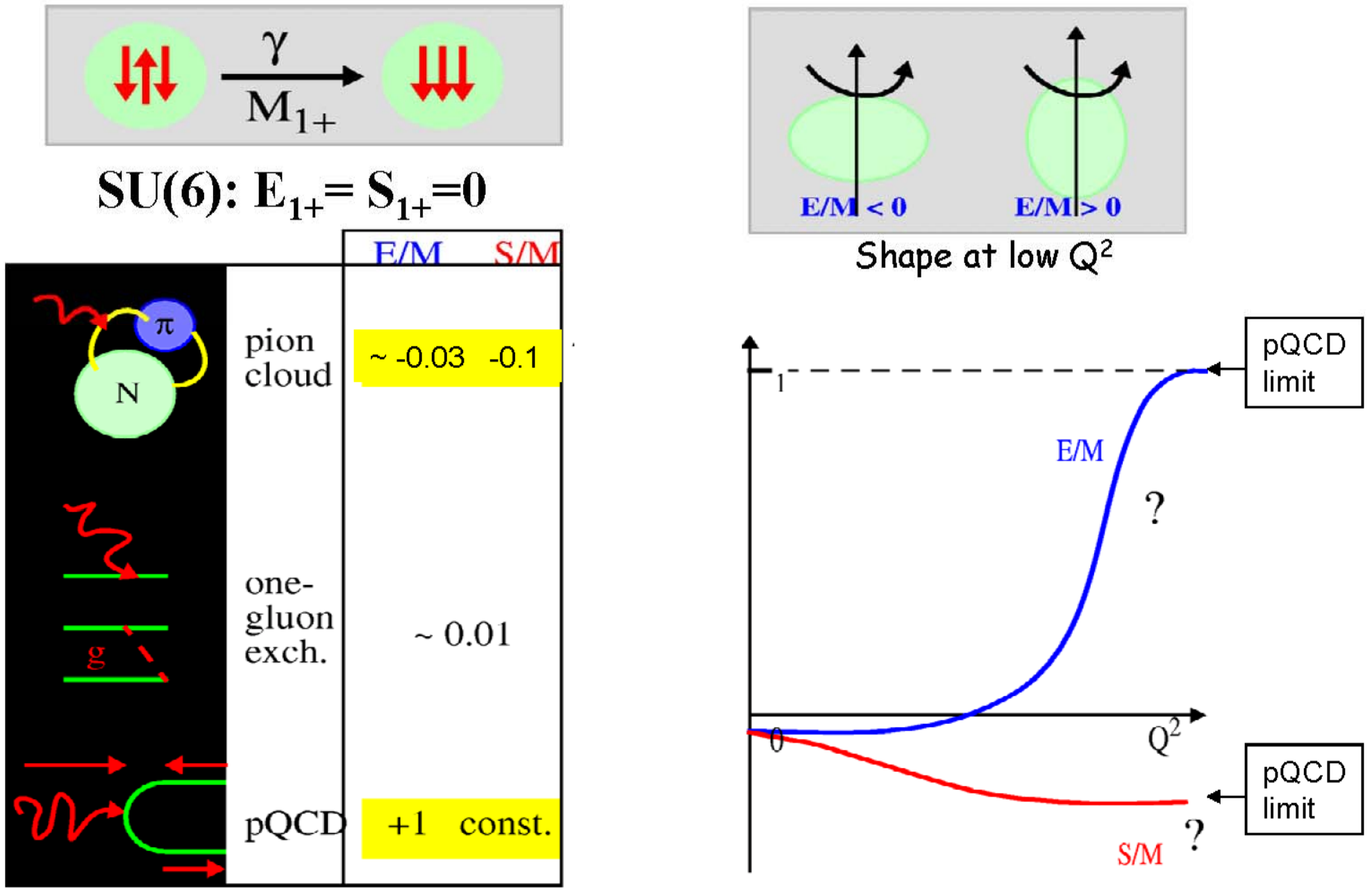


\section{$v^{\star} N \Delta$ Multipole Ratios $R_{E M}, R_{S M}$ before JLab}

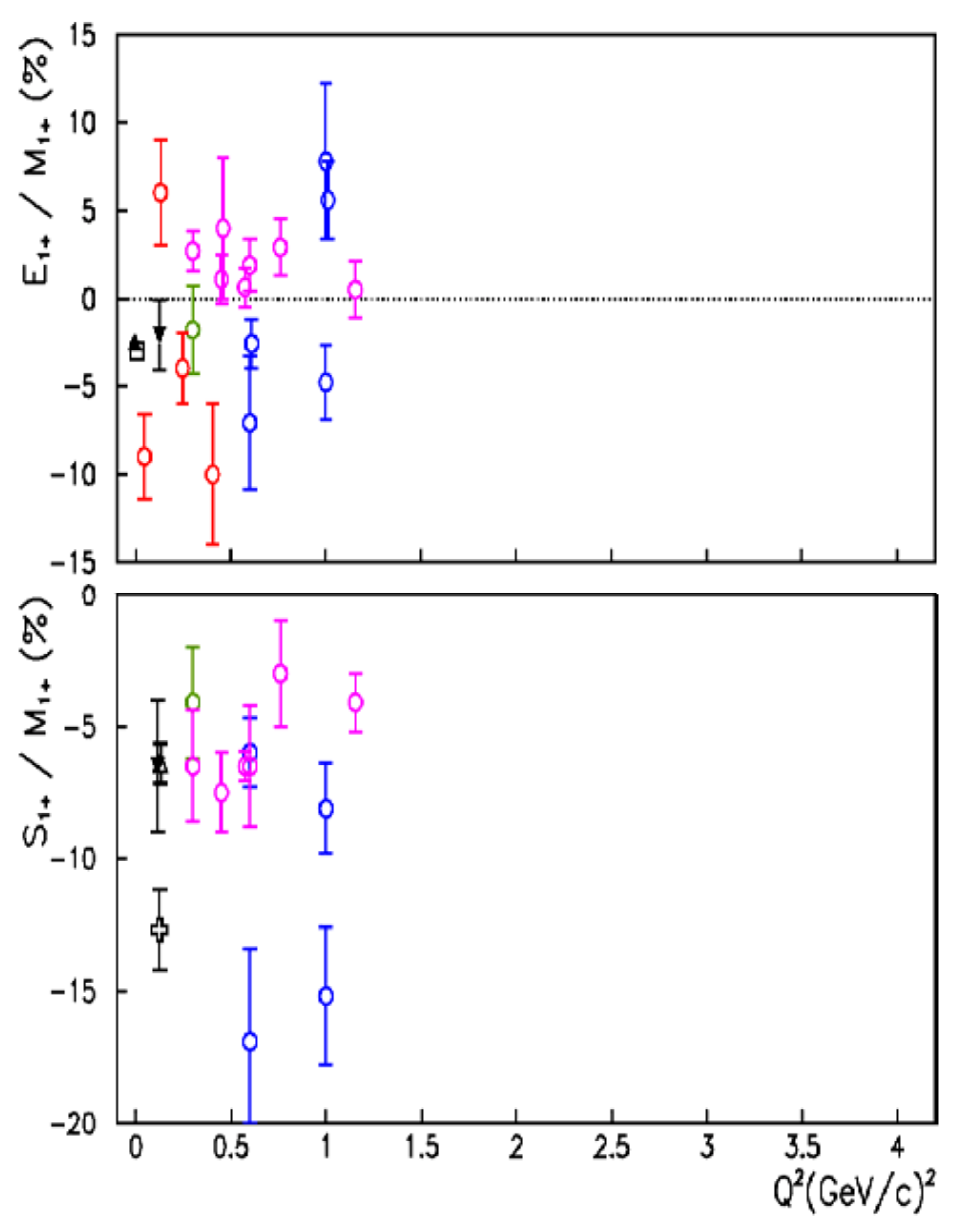

$$
\begin{aligned}
& R_{E M}=E_{1+} / M_{1+} \\
& R_{S M}=S_{1+} / M_{1+} \\
& =\text { Sign@ } Q^{2}>0 ? \\
& =Q^{2} \text { dependence? }
\end{aligned}
$$




\section{$\gamma^{*} N \Delta$ Multipole Ratios $R_{E M}, R_{S M}$ with JLab}

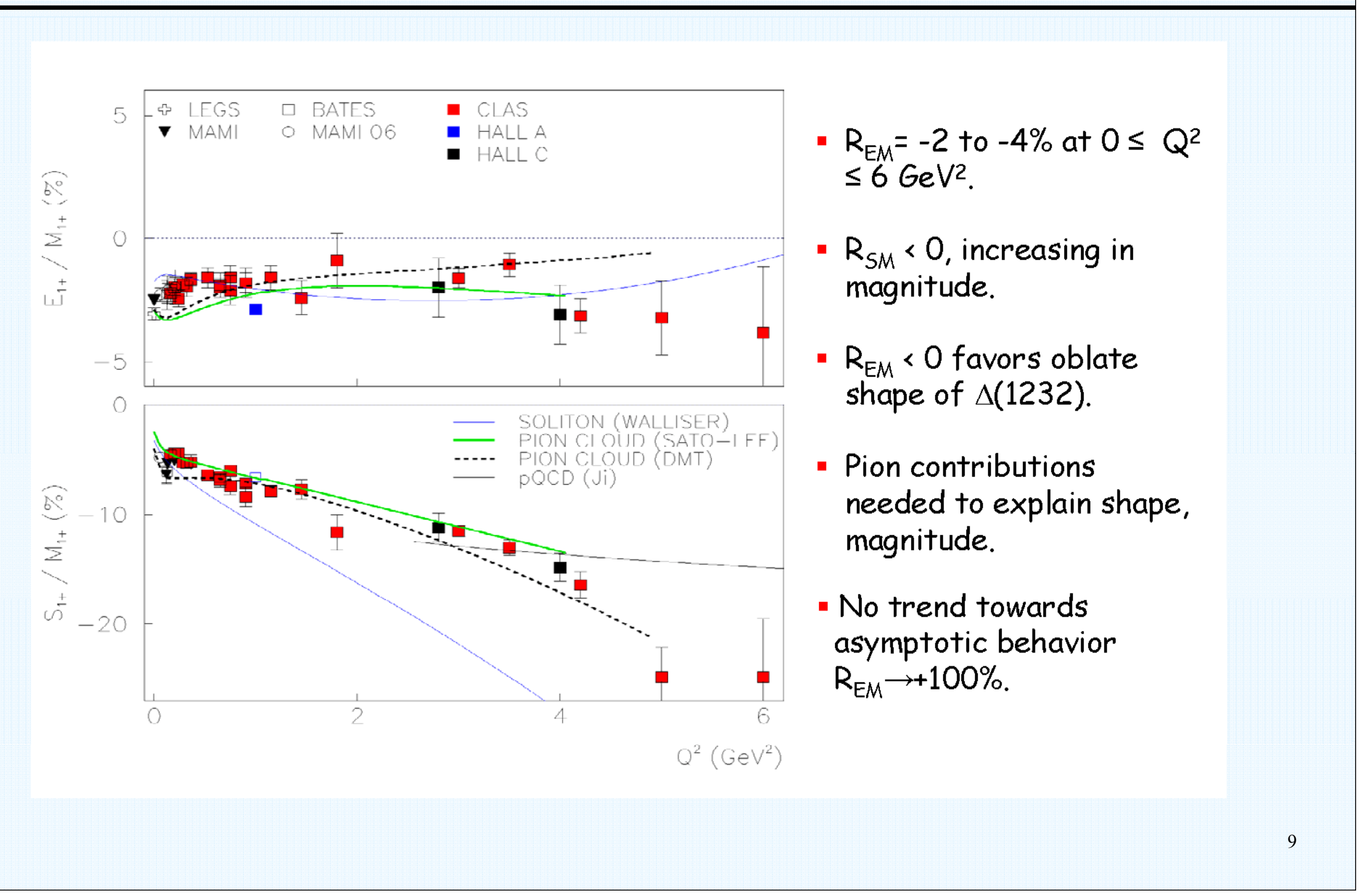




\section{$\mathrm{N}^{*}$ Transition Form Factors in the 2nd Resonance Region}

$P_{11}(1440)$ Poorly understood in nrCQMs.

Other models:

- Hybrid baryon with gluonic excitation $\left|q^{3} G\right\rangle$

- Quark core with large meson cloud $\left|q^{3} m\right\rangle$

- Nucleon-sigma molecule |No>

- Dynamically generated resonance

$\mathrm{S}_{11}(1535) \quad$ Hard form factor (slow fall off with $Q^{2}$ ) Not a quark resonance, but $K \Sigma$ dynamical system?

$D_{13}(1520)$ Change of helicity structure with increasing $Q^{2}$ from $\Lambda=3 / 2$
dominance to $\Lambda=1 / 2$ dominance, predicted in $n r C Q M s, p Q C D$. 


\section{Roper $P_{11}(1440)$ Helicity amplitudes}
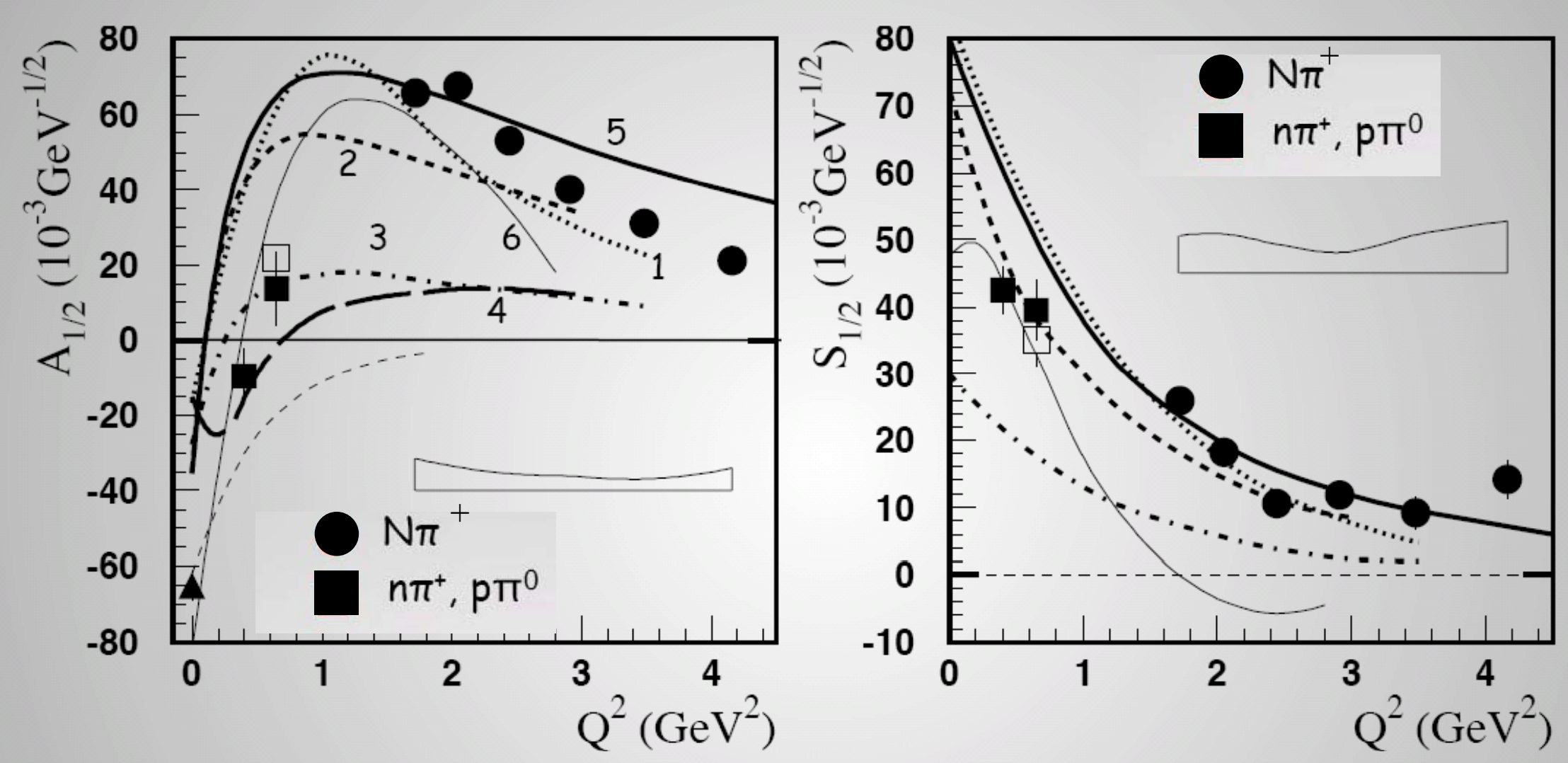

1. Weber, PRC41(1990)2783 2.Capstick, PRD51(1995)3598 3. Simula, PLB397 (1997)13 4.Riska, PRC69(2004)035212

5. Aznauryan, PRC76(2007)025212 6. Cano, PL B431(1998)270

I. G. Aznauryan et al. (CLAS), arXiv:080447 [nucl-ex] 


\section{Transverse amplitudes for $\gamma^{*} p \rightarrow>D_{13}(1520)$}

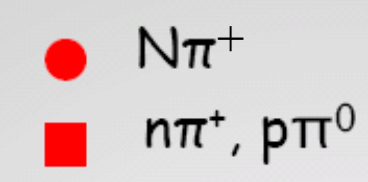

- M. Dugger et al.,

PRC76 025211, 2007
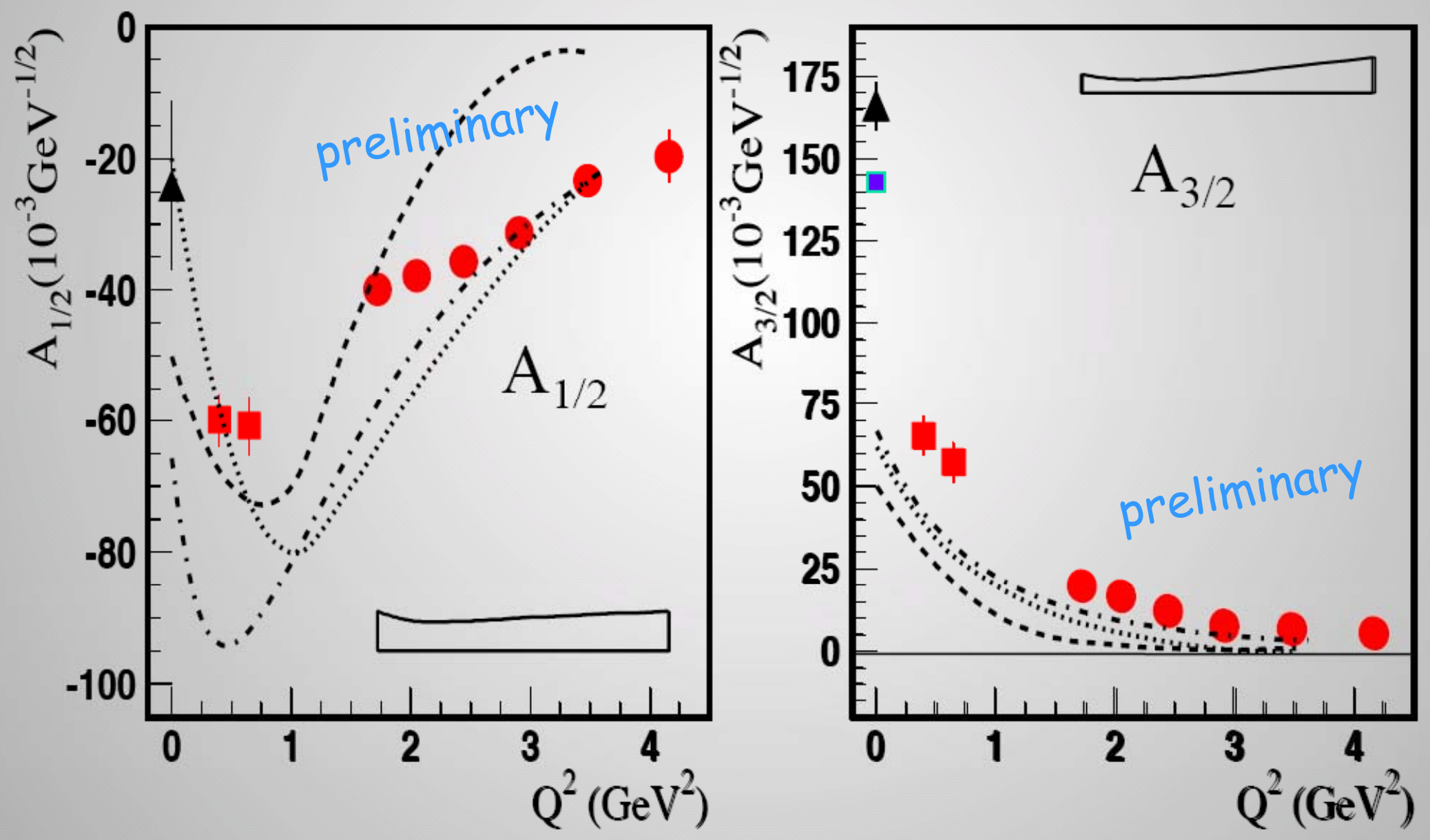


\section{CLAS Single Pion Electro-production Data}

\section{Data in the $\Delta(1232)$ region up to $\mathrm{W}=1.4 \mathrm{GeV}$}

\begin{tabular}{|l|l|l|l|l|}
\hline JLab/Hall C & Frolov & 1999 & $\mathrm{p} \pi^{0}$ & $\mathrm{Q}^{2}=2.5-4.3 \mathrm{GeV}^{2}$ \\
\hline Bates & Mertz et al. & 2001 & $\mathrm{p} \pi^{0}$ & $\mathrm{Q}^{2}=0.127 \mathrm{GeV}^{2}$ \\
\hline Mainz & Pospischil et al. & 2001 & $\mathrm{p} \pi^{0}$ & $\mathrm{Q}^{2}=0.127 \mathrm{GeV}^{2}$ \\
\hline JLab/CLAS & Joo et al. & 2002 & $\mathrm{p} \pi^{0}$ & $\mathrm{Q}^{2}=0.4-1.8 \mathrm{GeV}^{2}$ \\
\hline Bonn & Bantes, Gothe & 2002 & $\mathrm{p} \pi^{0}$ & $\mathrm{Q}^{2}=0.6 \mathrm{GeV}^{2}$ \\
\hline JLab/CLAS & Egiyan et al. & 2006 & $\mathrm{n} \pi^{+}$ & $\mathrm{Q}^{2}=0.3-0.6 \mathrm{GeV}^{2}$ \\
\hline Mainz & Elsner et al. / Stave et al. & 2006 & $\mathrm{p} \pi^{0}$ & $\mathrm{Q}^{2}=0.05-0.2 \mathrm{GeV}^{2}$ \\
\hline Jlab/CLAS & Ungaro et al. & 2006 & $\mathrm{p} \pi^{0}$ & $\mathrm{Q}^{2}=3.0-6.0 \mathrm{GeV}^{2}$ \\
\hline JLab/Hall A & Kelly et al. & 2007 & $\mathrm{p} \pi^{0}$ & $\mathrm{Q}^{2}=1.0 \mathrm{GeV}^{2}$ \\
\hline JLab/CLAS & Park et al. & 2008 & $\mathrm{n} \pi^{+}$ & $\mathrm{Q}^{2}=3.0-6.0 \mathrm{GeV}^{2}$ \\
\hline
\end{tabular}




\section{CLAS Single Pion Electro-production Data}

\section{Data up to the 3 rd resonance region up to $\mathrm{W}=1.7 \mathrm{GeV}$}

\begin{tabular}{|l|l|l|l|l|}
\hline JLab/CLAS & Joo et al. (A only) & 2003 & $\mathrm{p} \pi^{0}$ & $\mathrm{Q}^{2}=0.4-0.65 \mathrm{GeV}^{2}$ \\
\hline JLab/CLAS & Joo et al. (A $A_{e}$ only) & 2004 & $\mathrm{n} \pi^{+}$ & $\mathrm{Q}^{2}=0.4-0.65 \mathrm{GeV}^{2}$ \\
\hline JLab/Hall A & Laveissiere et al. & 2004 & $\mathrm{p} \pi^{0}$ & $\mathrm{Q}^{2}=1.0 \mathrm{GeV}^{2}$ \\
\hline JLab/CLAS & Egiyan et al. & 2006 & $\mathrm{n} \pi^{+}$ & $Q^{2}=0.3-0.6 \mathrm{GeV}^{2}$ \\
\hline JLab/CLAS & Park et al. & 2008 & $\mathrm{n} \pi^{+}$ & $Q^{2}=1.7-4.5 \mathrm{GeV}^{2}$ \\
\hline
\end{tabular}




\section{CLAS Single $\pi^{0}$ Electro-production Data Analysis}

- E1E:

- Beam Energy: $1 \mathrm{GeV}$ and $2 \mathrm{GeV}$

- Low $\mathrm{Q}^{2}$ up to $1.5 \mathrm{GeV}$, and $\mathrm{W}$ up to $1.7 \mathrm{GeV}$

-E1-6:

- Beam Energy: $5.75 \mathrm{GeV}$

- Low $\mathrm{Q}^{2}$, up to $4.5 \mathrm{GeV}$ and $\mathrm{W}$ up to $2.0 \mathrm{GeV}$

- Beam polarization: 70-80\%

- Target: Liquid Hydrogen 


\section{CEBAF at Jefferson Lab}

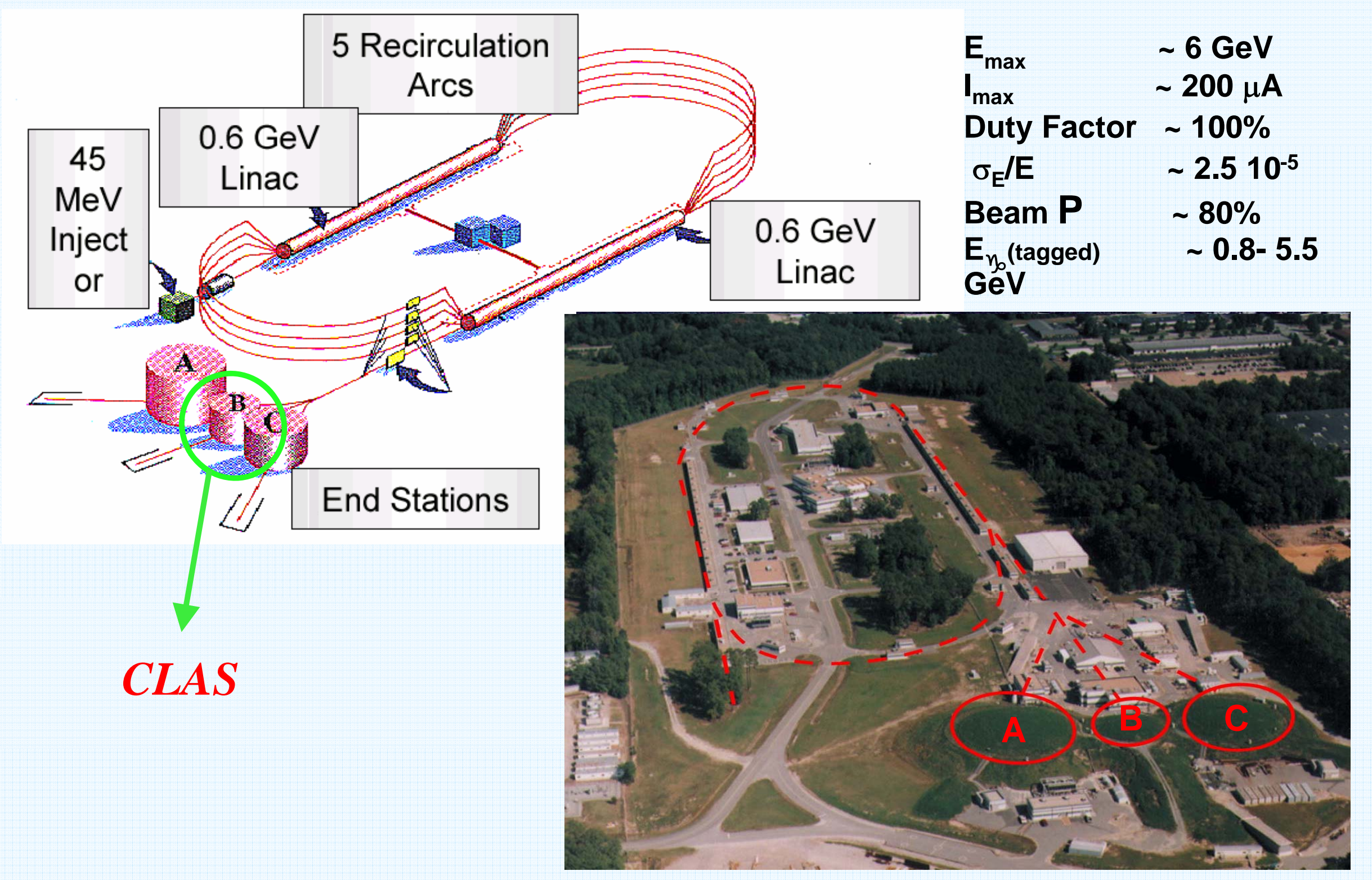




\section{CEBAF Large Acceptance Spectrometer (CLAS)}

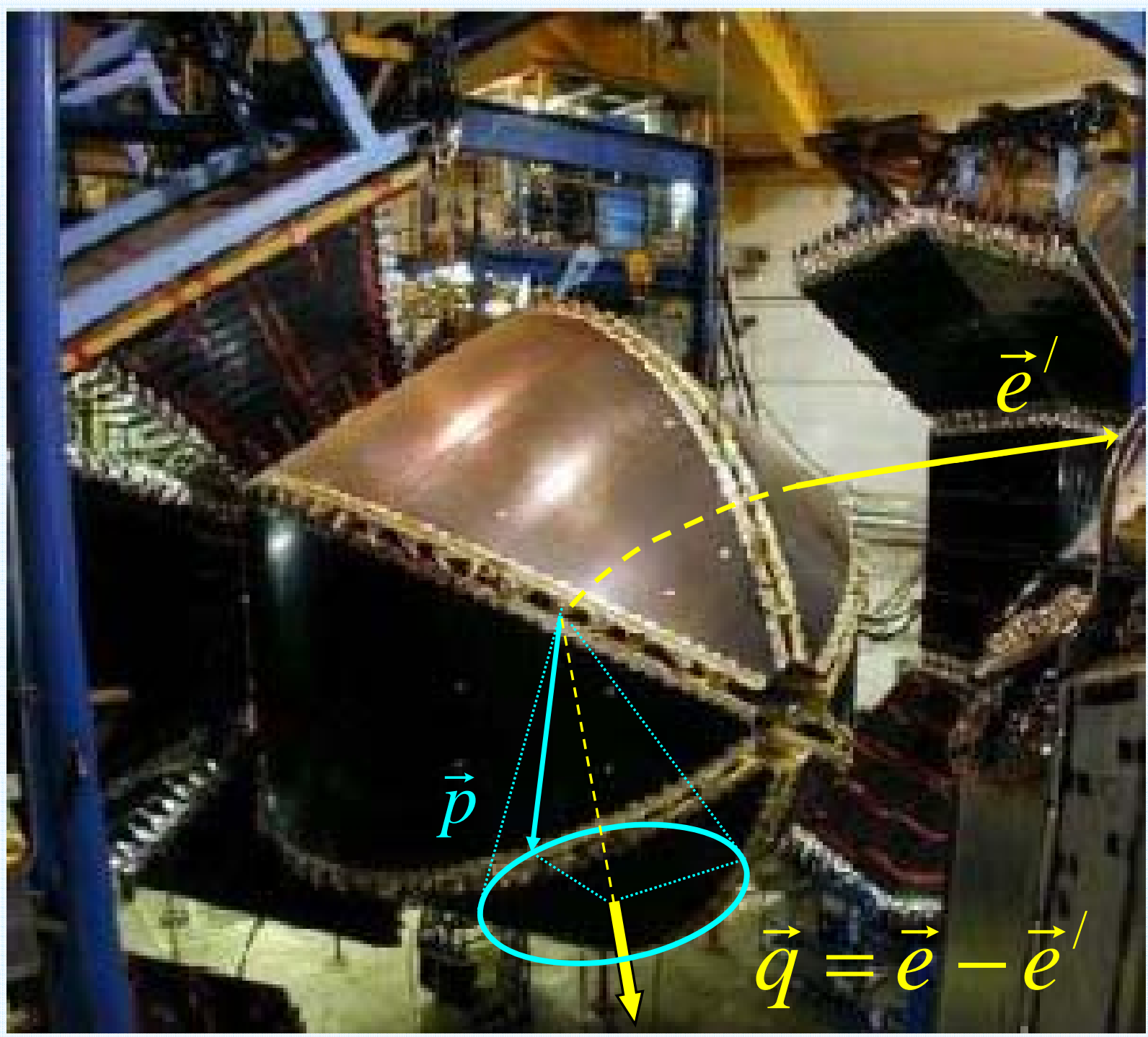

- Six identical sectors

. 5 T toroidal B-field

- $\Delta \theta=15-140$ degrees

- $\Delta \varphi=0-50$ degrees

$\cdot \Delta p / p=10^{-2}-10^{-3}$ 


\section{Electron PID}

- Number of photo-electrons ( $n p h e$ ) in the Cerenkov detector

- EC Threshold

- EC Sampling Fraction

- Track Coordinates in the EC plane

- Minimum Ionizing Particles rejection

- Electromagnetic Shower Shape 


\section{Electron PID}

$10 \times$ Number of photo-electrons

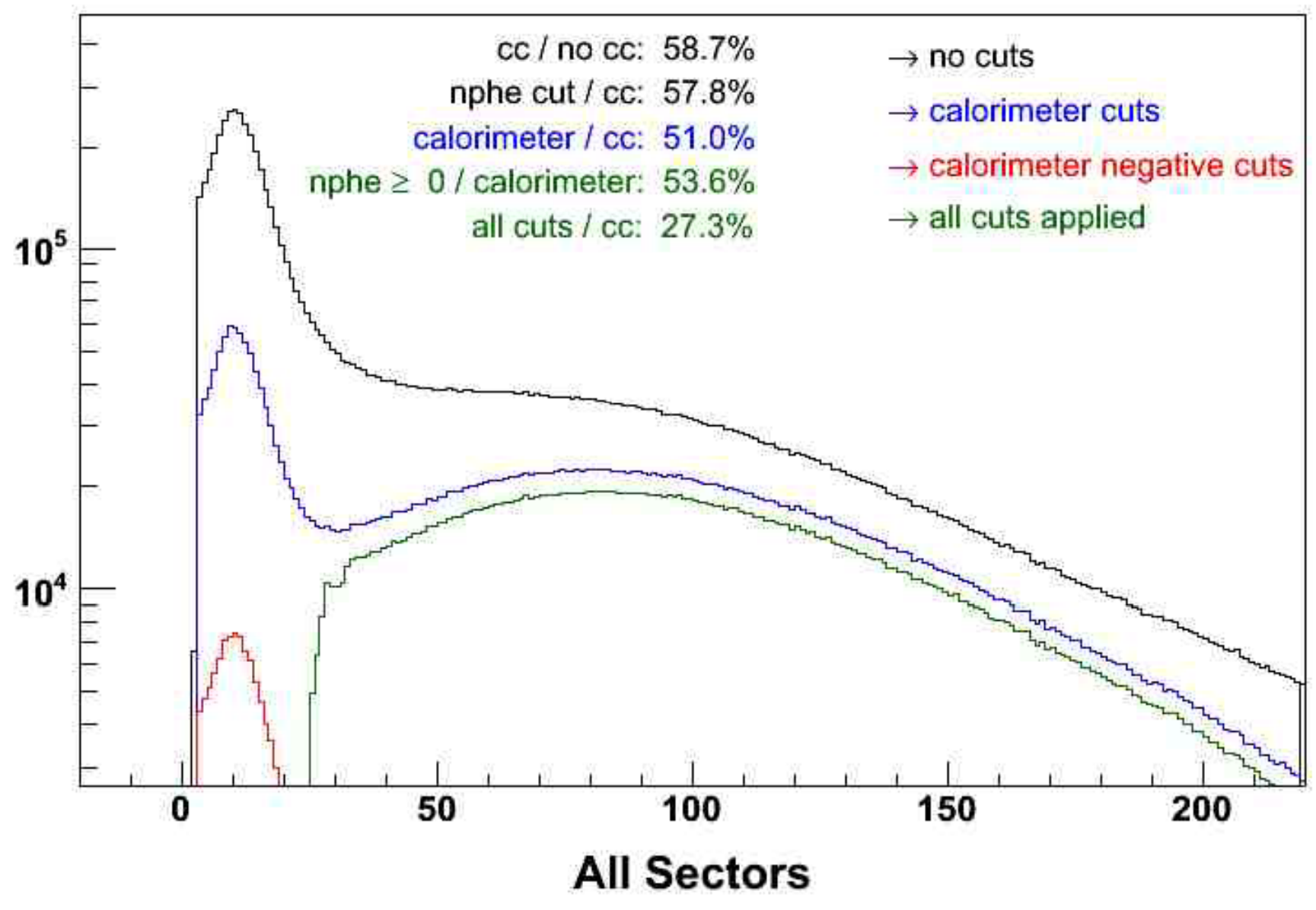




\section{Proton PID}

Use TOF timing and DC momentum of positive tracks

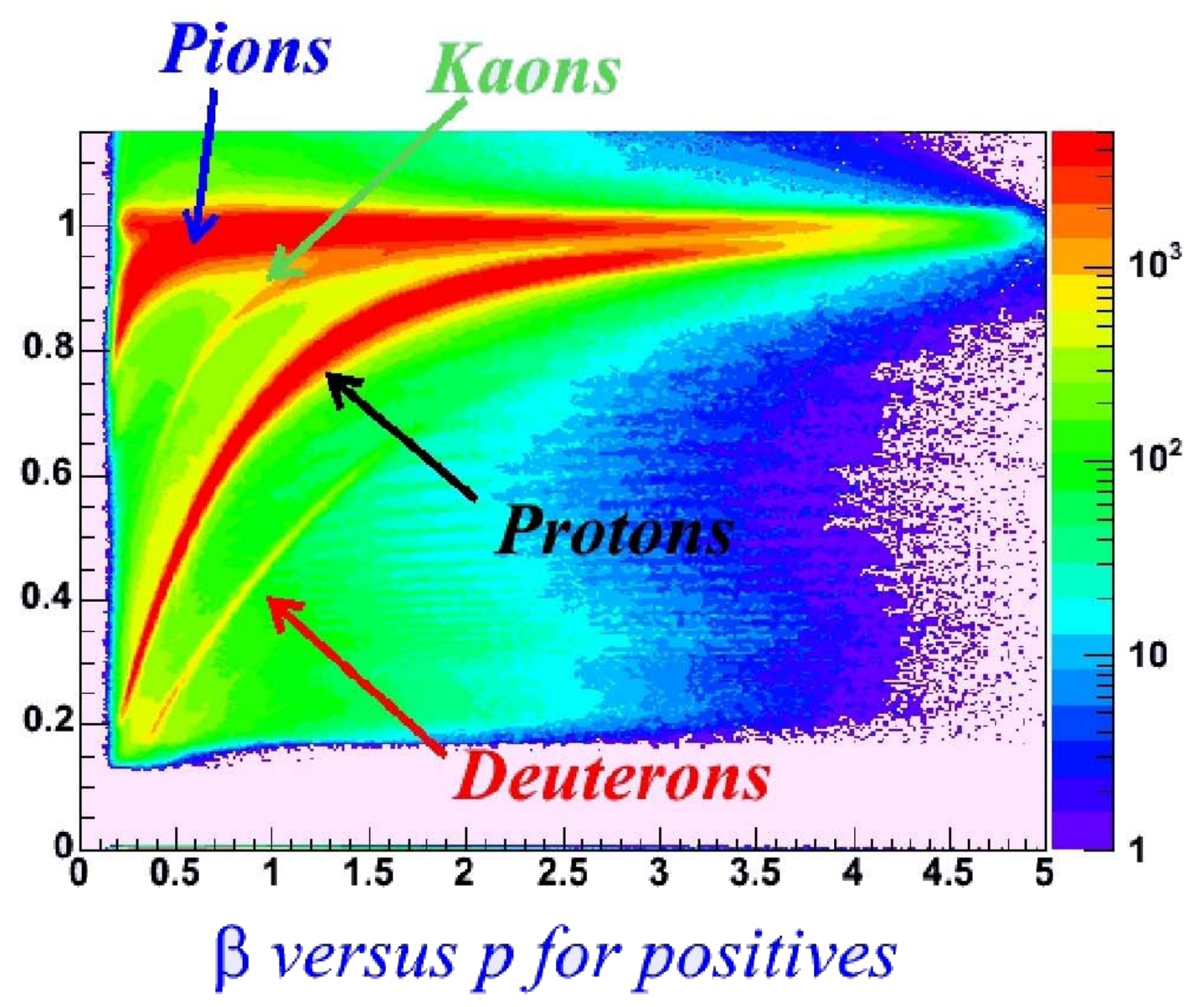




\section{$\pi^{0}$ selection}

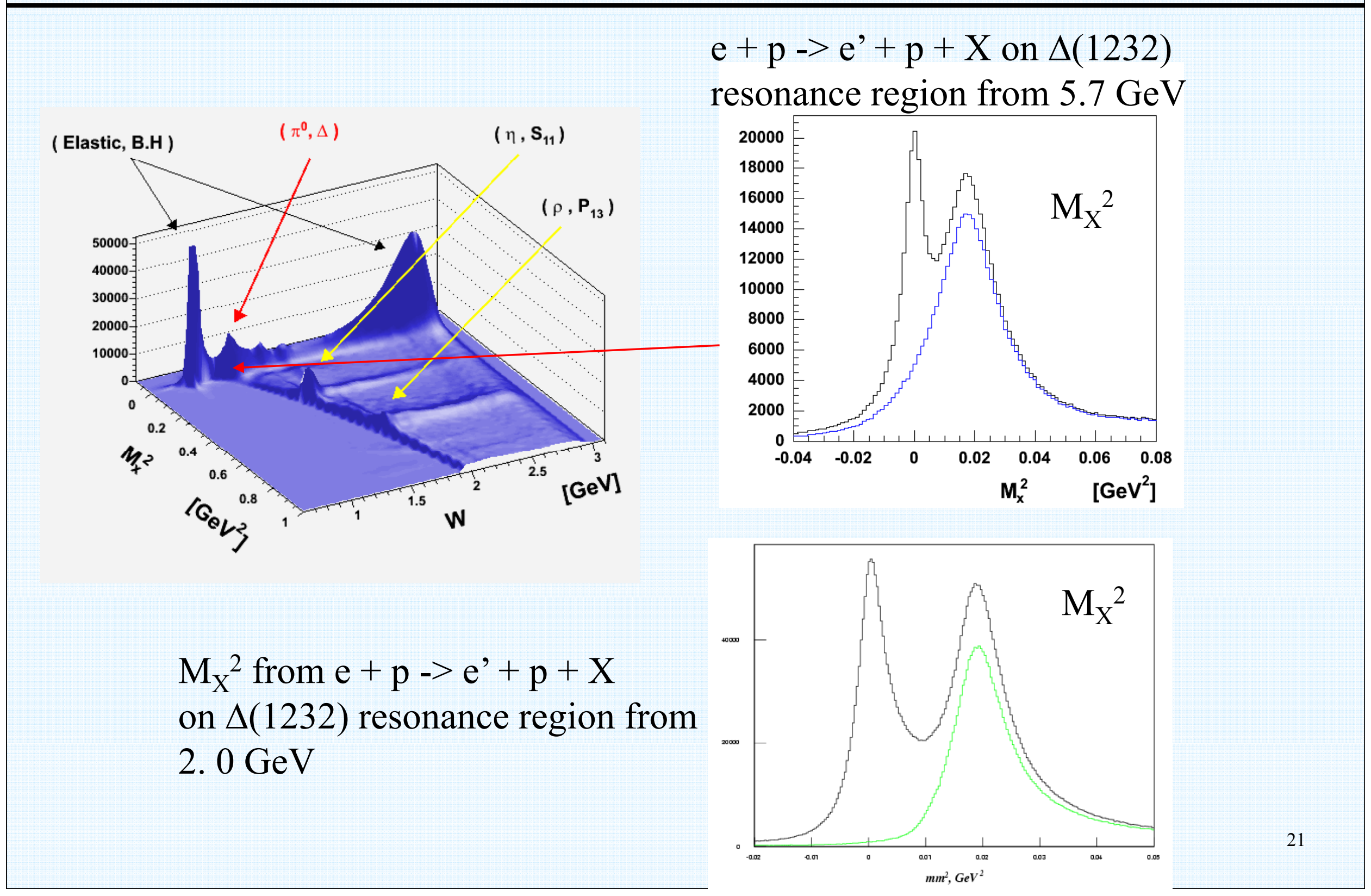




\section{$\pi^{0}$ selection (E1-6)}

$$
\begin{gathered}
\mathrm{M}_{\mathrm{X}}^{2} \text { from } \mathrm{e}+\mathrm{p}->\mathrm{e}^{\prime}+\mathrm{p}+\mathrm{X} \\
\text { for } 1.1<\mathrm{W}<2.0 \mathrm{GeV}
\end{gathered}
$$

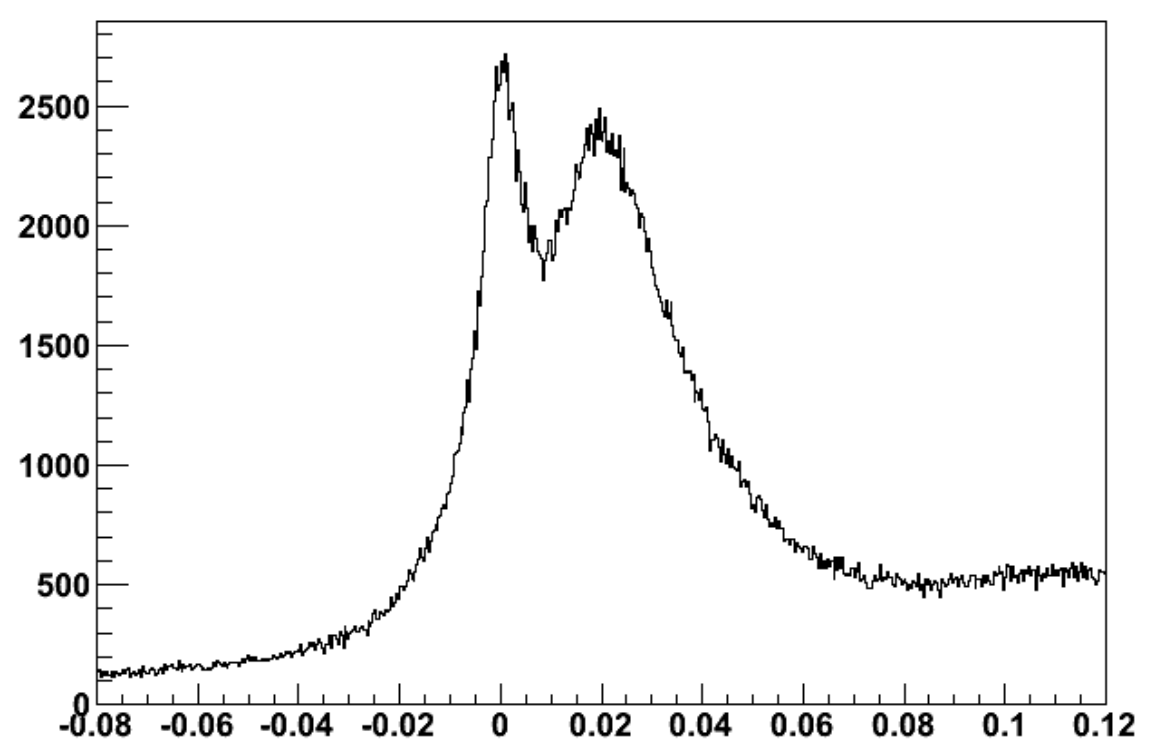

Currently working on bench-mark studies on $\Delta(1232)$ region with/without tagging $\pi^{0}$

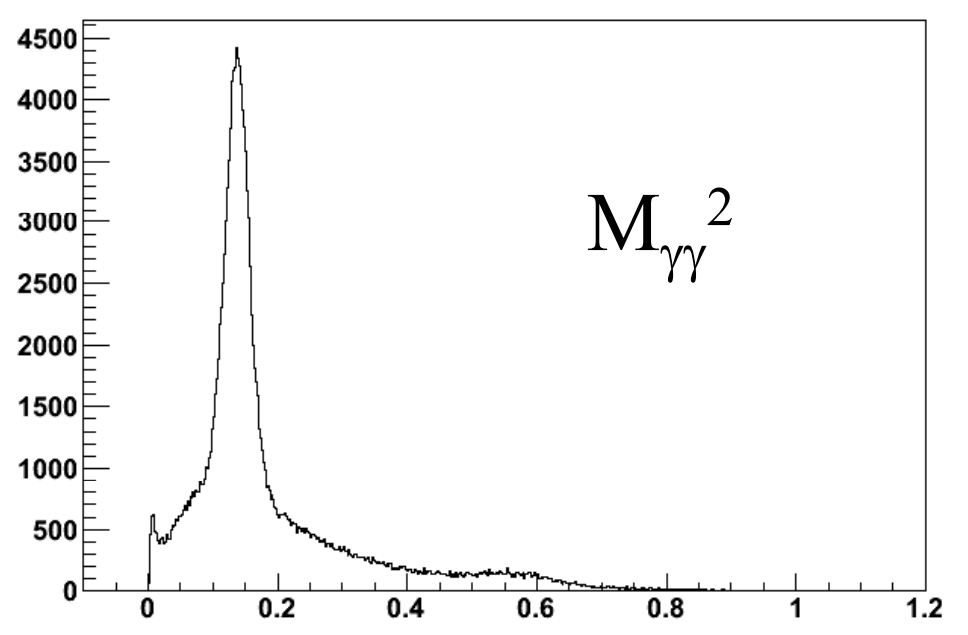

Entries 190785

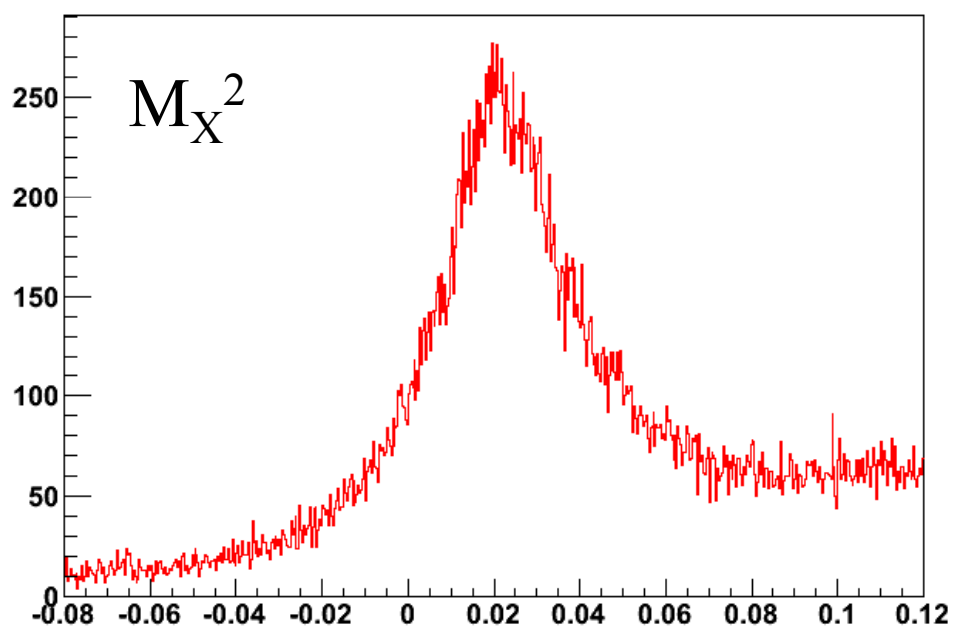




\section{Typical ep $\rightarrow e^{\prime} p \pi^{0}$ cross sections vs $\cos \theta^{*}$ and $\varphi^{*}$}

$$
\mathrm{Q}^{2}=0.2 \mathrm{GeV}^{2} \mathrm{~W}=1.22 \mathrm{GeV}
$$
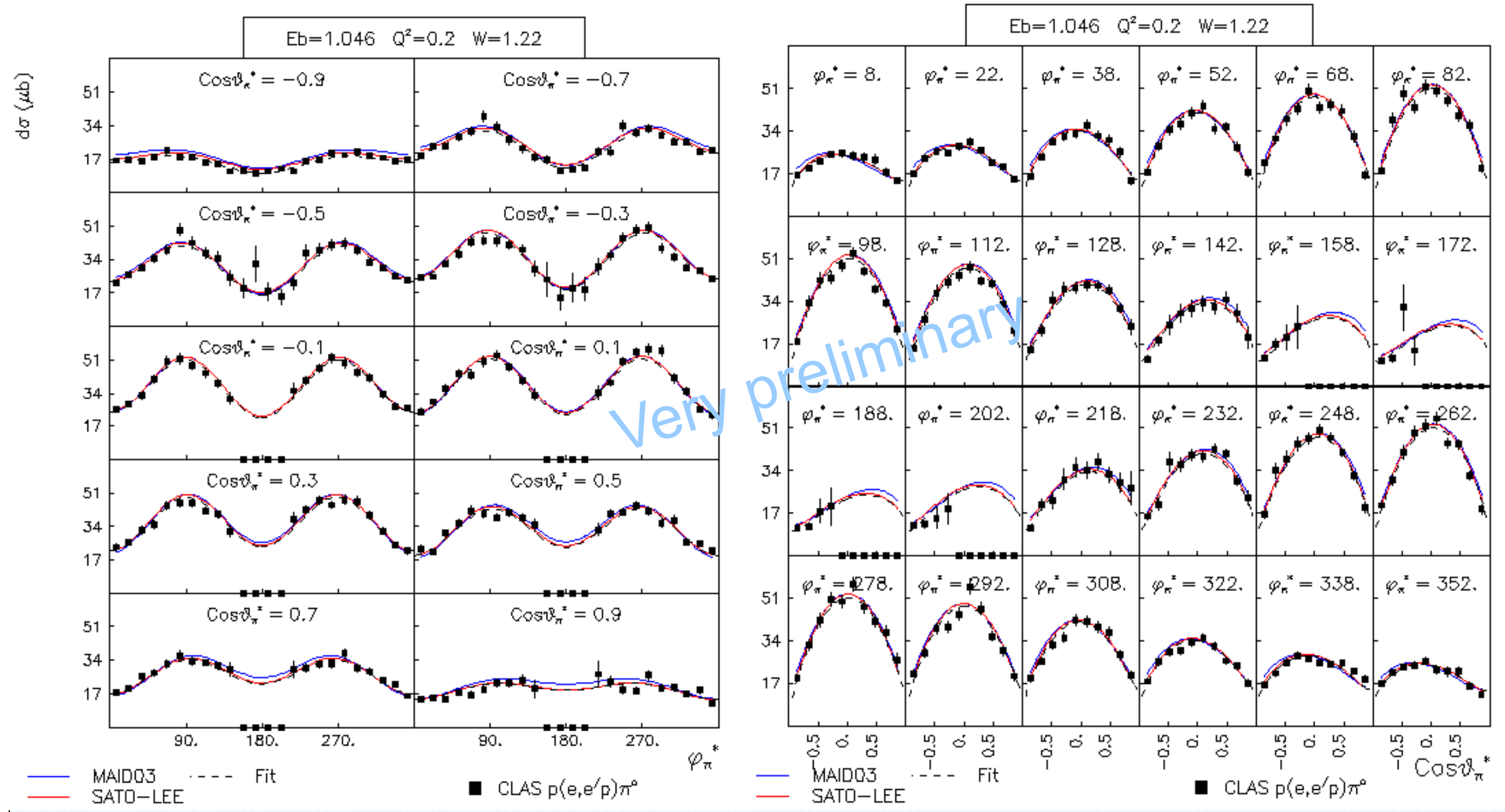


\section{$\Pi^{+}$electroproduction at $\mathrm{Q}^{2}=0.20 \mathrm{GeV}^{2}$ using CLAS}

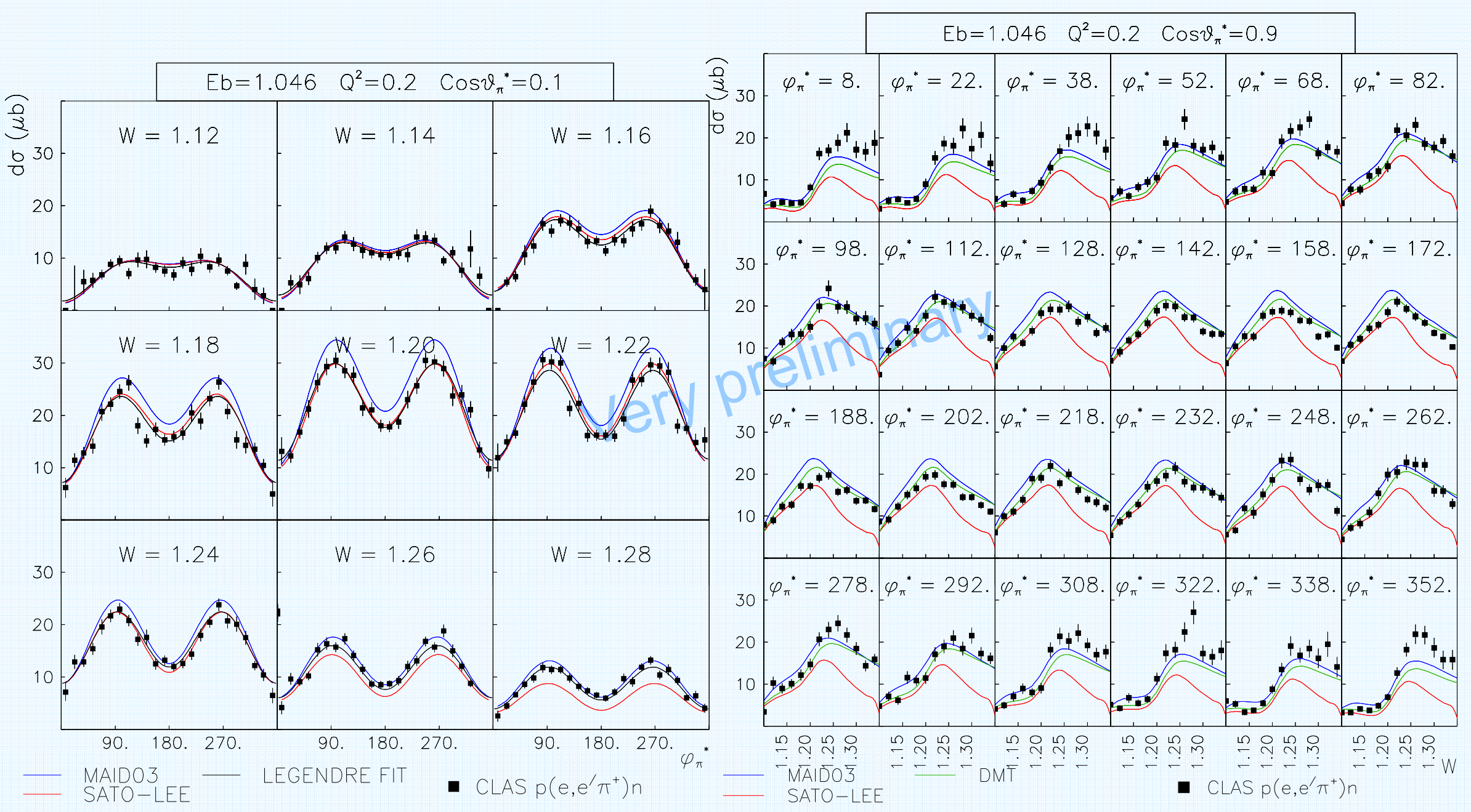




\section{Differential Cross section from $2.0 \mathrm{GeV}$}

$$
0.6<\mathrm{Q}^{2}<0.7,1.3<\mathrm{W}<1.35
$$
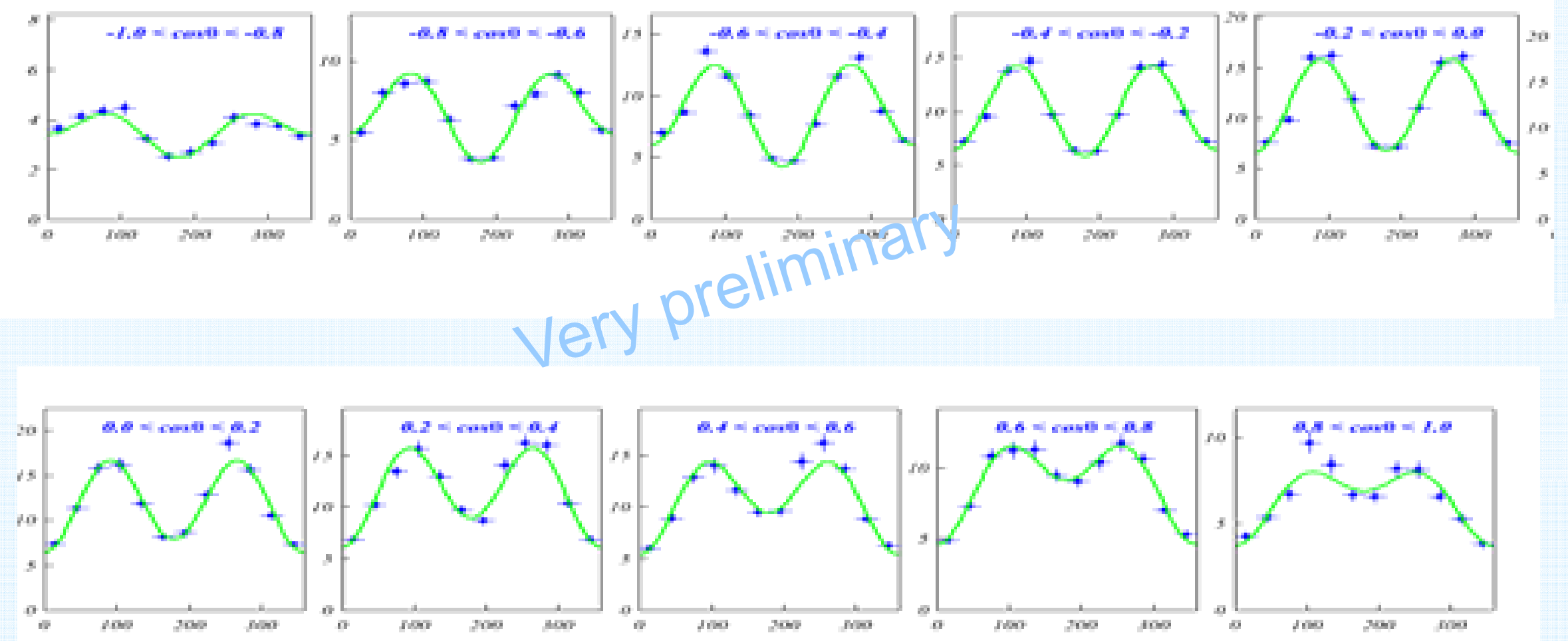


\section{Legendre Moment}

$$
\sigma_{T}+\varepsilon \sigma_{L}=\sum_{i=0}^{i=2} A_{i} P_{i}(\cos \theta)
$$

$0.6<\mathrm{Q} 2<0.7$

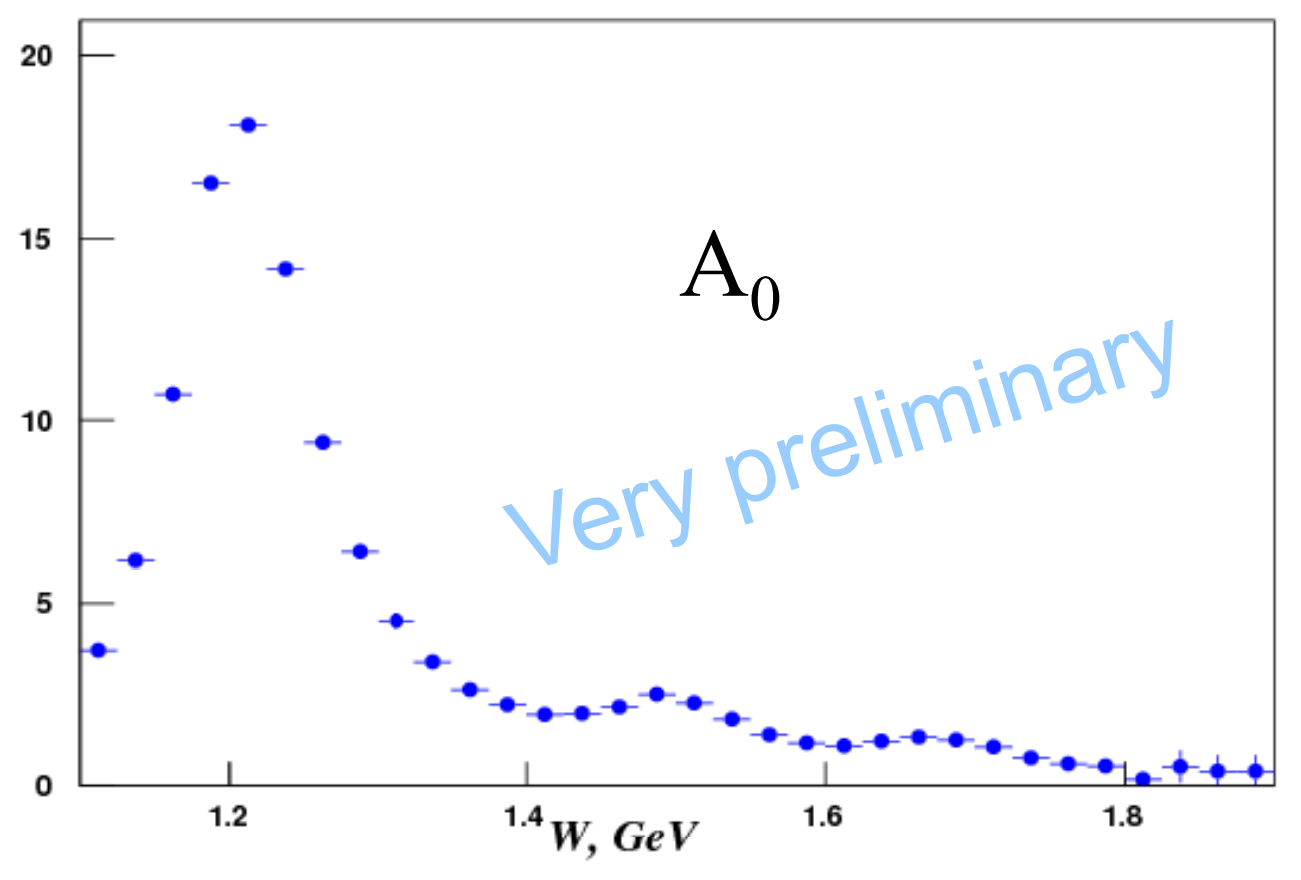




\section{$\mathrm{E} 1+/ \mathrm{M} 1+$ and $\mathrm{S} 1+/ \mathrm{M} 1+$}

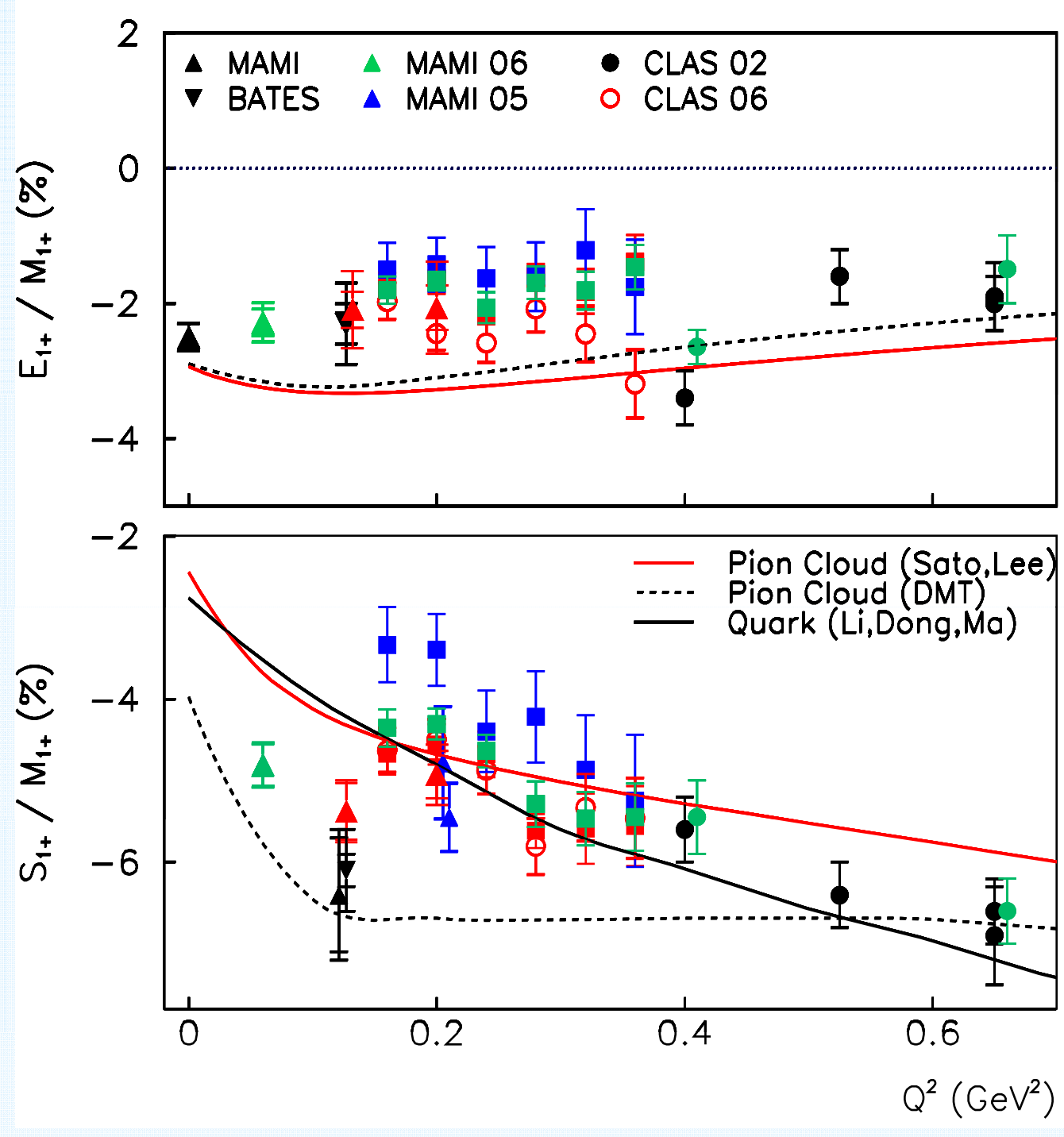

\section{CLAS e1e UIM Fit}

$\square \quad \mathrm{p} \pi^{0}$ only

$\square \quad n \pi^{+}$only

$\square \quad \mathrm{p} \pi^{0}$ and $n \pi^{+}$ 


\section{$\gamma^{*} N \Delta$ Multipole Ratios $R_{E M}, R_{S M}$ with JLab}

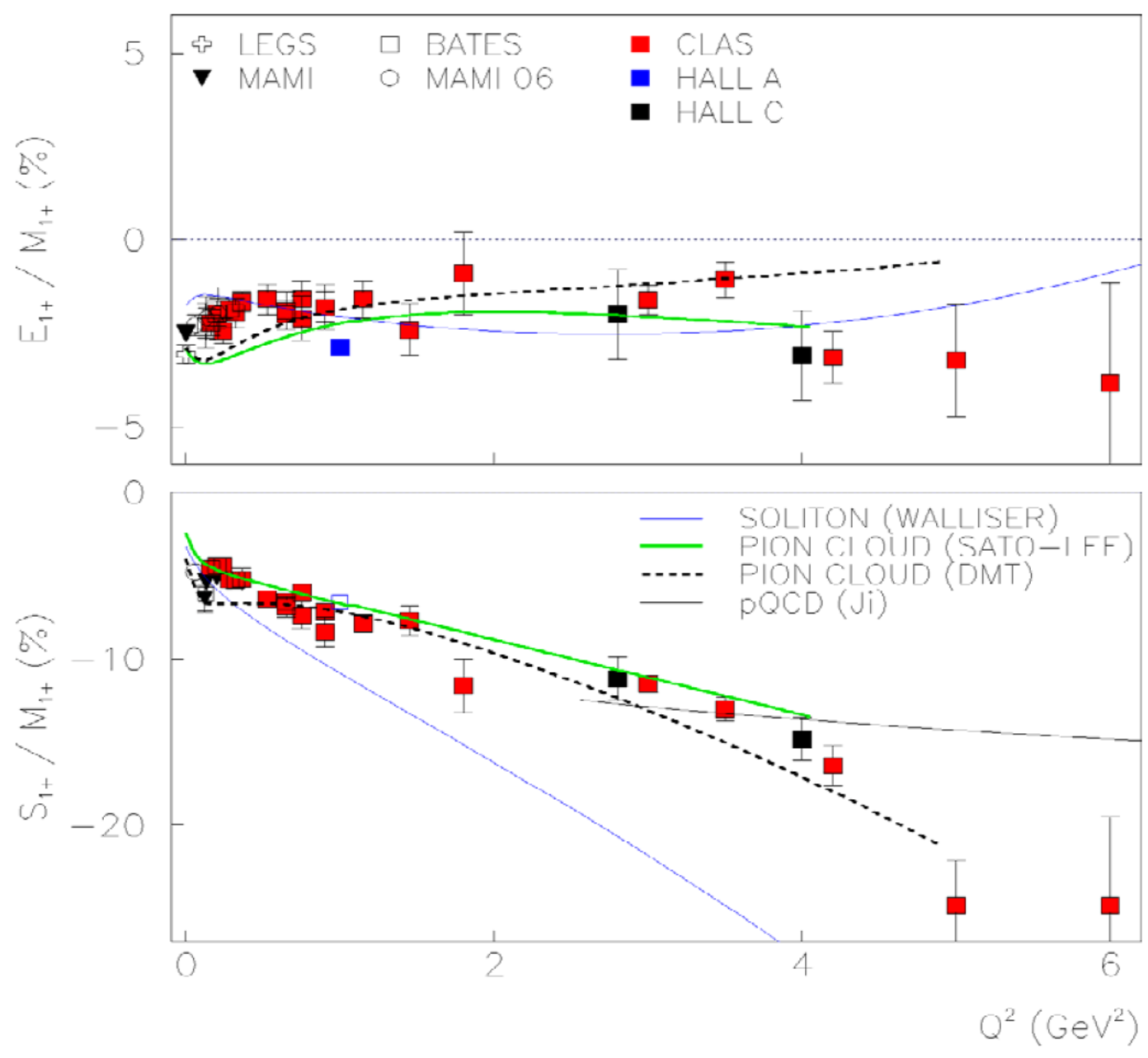

- $R_{E M}=-2$ to $-4 \%$ at $0 \leq Q^{2}$ $\leq 6 \mathrm{GeV}^{2}$.

- $R_{S M}<0$, increasing in magnitude.

- $R_{E M}<0$ favors oblate shape of $\Delta(1232)$.

- Pion contributions needed to explain shape, magnitude.

- No trend towards asymptotic behavior $R_{E M} \rightarrow+100 \%$. 


\section{Roper $\mathrm{P}_{11}(1440)$ Helicity amplitudes}
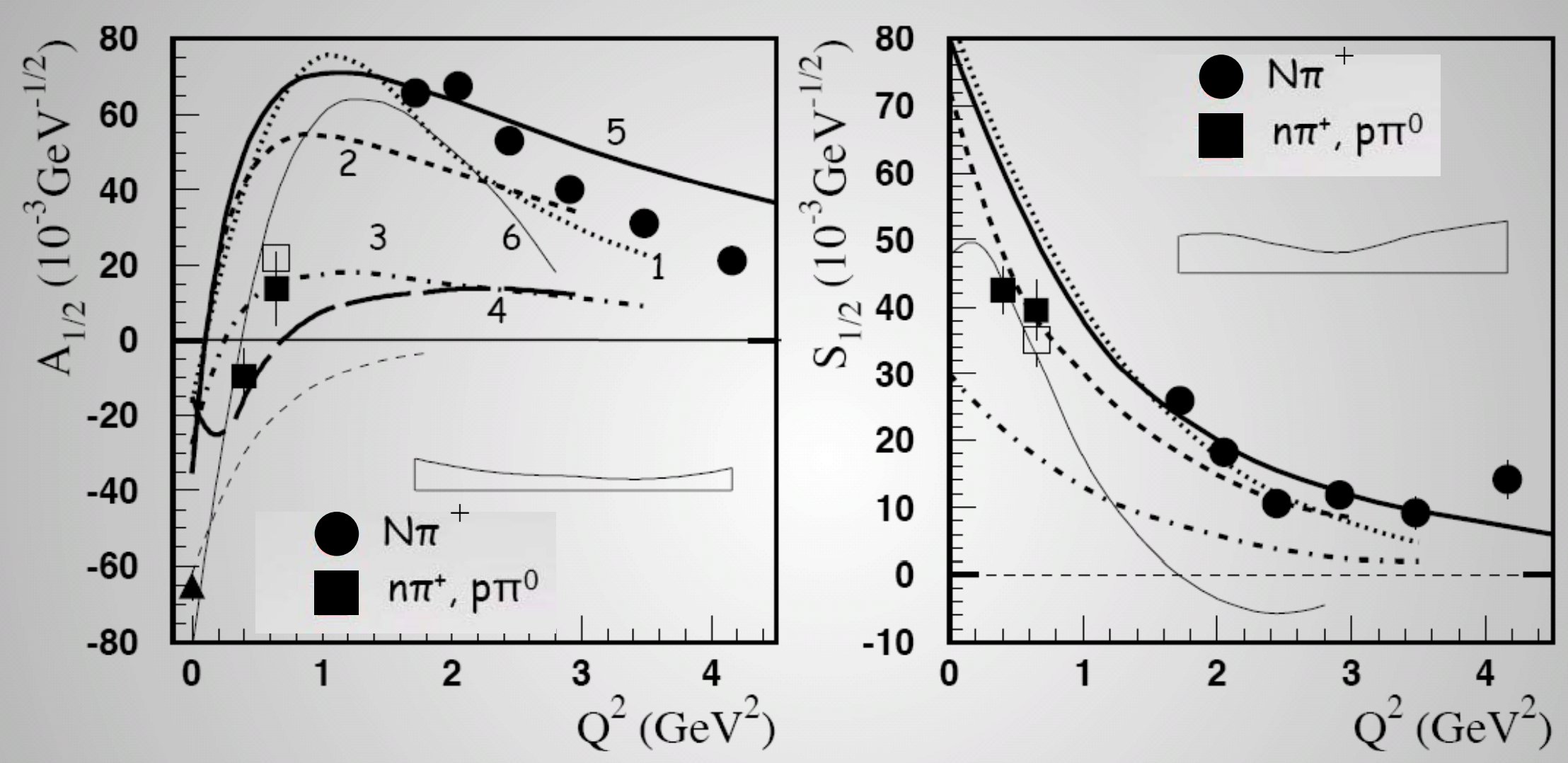

1. Weber, PRC41(1990)2783 2.Capstick, PRD51(1995)3598

3. Simula, PLB397 (1997)13 4.Riska, PRC69(2004)035212

5.Aznauryan, PRC76(2007)025212 6. Cano, PL B431(1998)270

I. G. Aznauryan et al. (CLAS), arXiv:080447 [nucl-ex] 


\section{Transverse amplitudes for $\gamma^{*} p->D_{13}(1520)$}

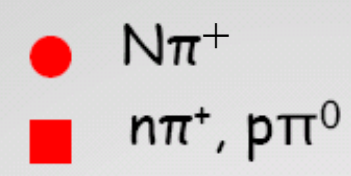

- M. Dugger et al., PRC76 025211, 2007
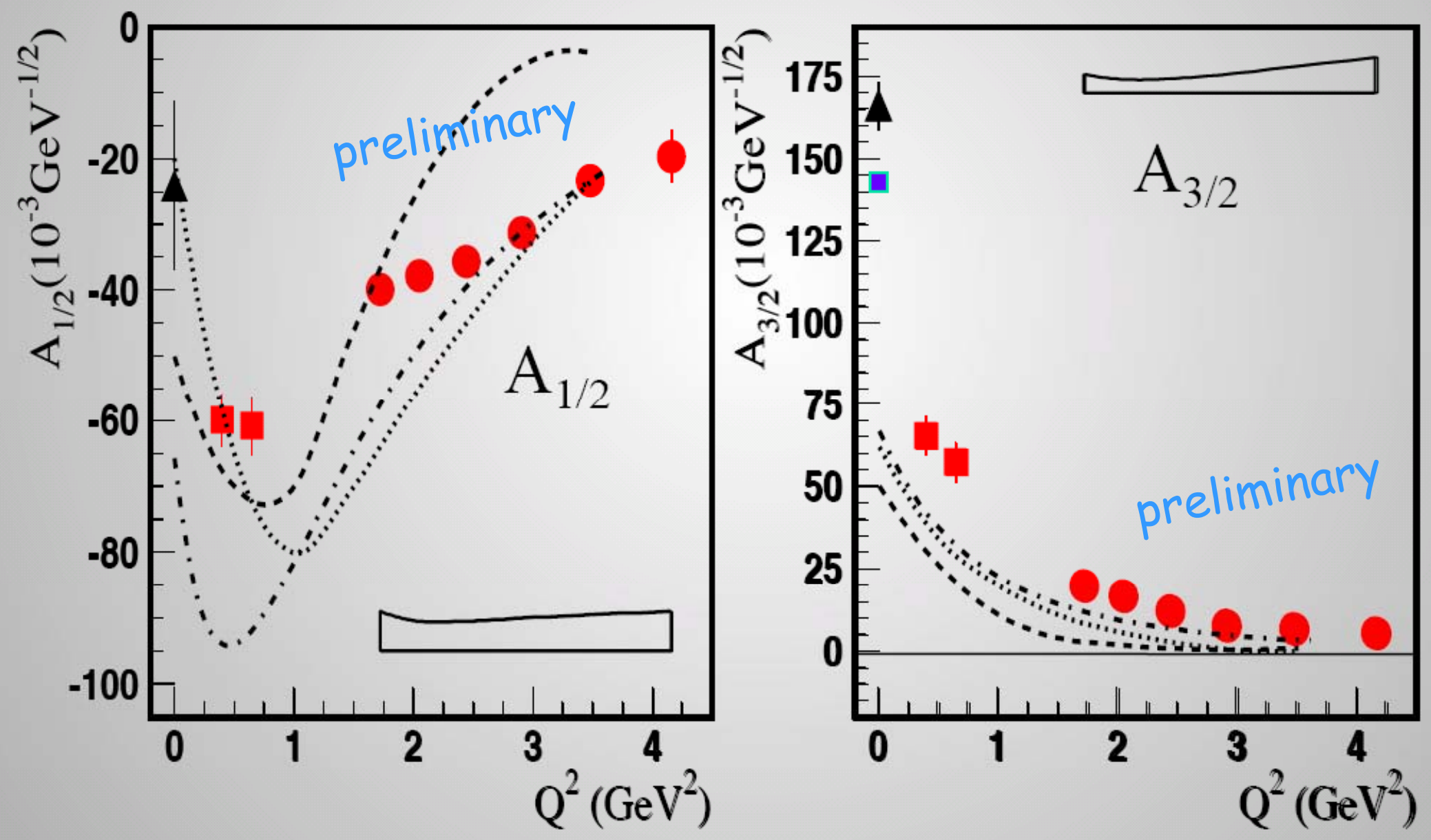


\section{CLAS12 - Detector}

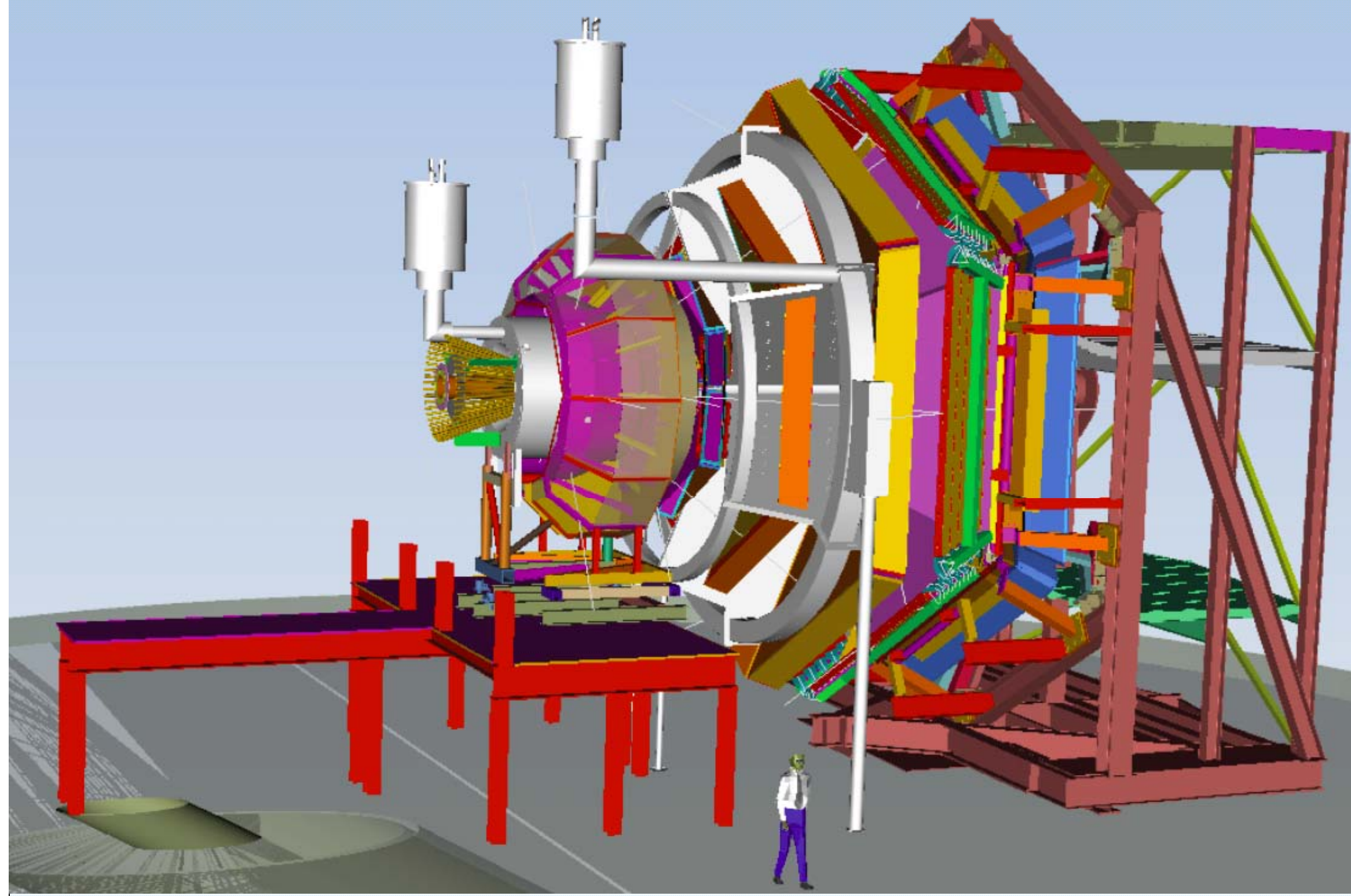




\section{Projections for $\mathrm{N}^{*}$ Transition Amplitudes@12 GeV}
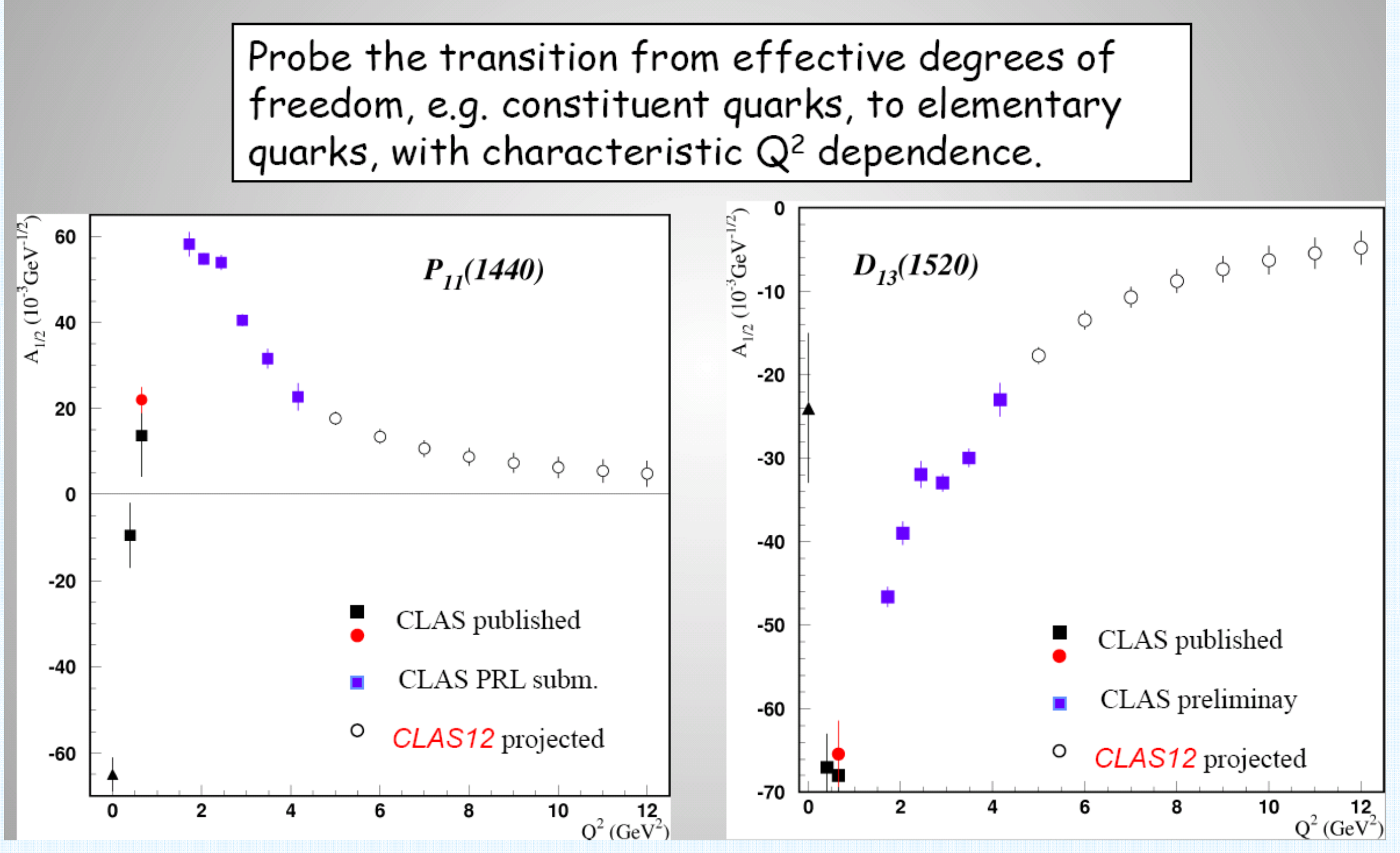


\section{Summary}

- Single $\pi^{0}$ electro-production analysis is under way for low and high $\mathrm{Q}^{2}$ range up to $2.0 \mathrm{GeV}$ in $\mathrm{W}$.

- Able to perform combined analysis with $n \pi^{+}$and two pion channels

- $\mathrm{p} \pi^{0}$ channel is an important part of $12 \mathrm{GeV} \mathrm{N}$ * program with CLAS12.

- Current $\mathrm{p} \pi^{0}$ analysis measuring all final states will provide an important step for $12 \mathrm{GeV} \mathrm{N}^{*}$ program. 